\title{
Original synthesis route of bulk binary superconducting graphite intercalation compounds with strontium, barium and ytterbium
}

\author{
S. Cahen, ${ }^{* a}$ I. El-Hajj, ${ }^{\text {a } L . ~ S p e y e r, ~}{ }^{\text {a }}$ P. Berger, ${ }^{b}$ G. Medjahdi, ${ }^{\text {a } P . ~ L a g r a n g e, ~}{ }^{\text {a }}$ G. Lamura ${ }^{\mathrm{c}}$ and C. Hérold ${ }^{\mathrm{a}}$ \\ Thanks to its lamellar structure, graphite can host various chemical species between its interplanar spaces to form Graphite \\ Intercalation Compounds (GIC). Intercalation of metals into graphite can lead to remarkable modifications of the physical \\ properties of the sample with respect to pristine graphite as for instance superconductivity. In this paper, we report attempts \\ made to intercalate strontium, baryum and ytterbium into graphite, using a recent method involving a $\mathrm{LiCl}-\mathrm{KCl}$ molten salt \\ as a reaction medium. Resulting samples are characterized regarding their structure and chemical composition. We reveal \\ the obtaining of bulk $\mathrm{SrC}_{6}$ and $\mathrm{BaC}_{6}$ and a superficial intercalation of ytterbium leading to superconducting $\mathrm{YbC}_{6}$ domains \\ only, as confirmed by dc-magnetization measurements.
}

\section{Introduction}

Graphite is a stacking of graphene layers tied amongst themselves by means of weak van der Waals's bonds. In these layers, the carbon atoms are $\mathrm{sp}^{2}$ hybridized and each one is linked to three other atoms using strong covalent bonds. Consequently, the symmetry of the layers is hexagonal, creating a honeycomb 2D structure. ${ }^{1}$

Thanks to this significant structural anisotropy, graphite appears as a remarkable host material able to carry out numerous topotactic intercalation reactions. ${ }^{2}$ In order to proceed, the reactive species opens the 2D galleries and intercalates inside by means of an electron transfer between graphene and reagent, according to an oxido-reduction reaction. ${ }^{3}$

The intercalation can be partial or on the contrary complete, according to the considered cases. ${ }^{4}$ When the reaction is partial, the intercalated species occupies regularly one gallery only in $s$. This $s$ number characterizes the "stage" of the intercalation compound. When the reaction is complete, all the galleries are occupied so that the compound belongs to the stage 1 .

In this paper, we are interested in the intercalation of various pure metals into graphite. The oldest synthesis method, which was largely used, is also the cleanest. It consists in a reaction between solid graphite and metal in the vapour phase at moderate temperature, carried out inside a sealed tube under high vacuum. ${ }^{5}$

The electron transfer to the graphene planes is particularly easy in the case of the strongly electropositive elements as alkali

\footnotetext{
a. Institut Jean Lamour - UMR 7198 CNRS-UL - 2 Allée André Guinier, B.P. 50840, 54011 Nancy Cedex, France.

b. NIMBE, CEA, CNRS, Université Paris-Saclay, CEA Saclay, 91191, Gif sur Yvette Cedex, France.

c. CNR-SPIN, Corso Perrone 24, 16152, Genova, Italy.
}

metals, and especially potassium, rubidium and cesium..$^{5-7}$ Moreover, these metals present a significant vapour pressure even at low temperature. Thanks to this combination of properties, the three heavy alkali metals intercalate very easily into graphite, leading among others to first stage Graphite Intercalation Compounds (GIC) whose chemical formula writes $\mathrm{MC}_{8}(\mathrm{M}=\mathrm{K}, \mathrm{Rb}, \mathrm{Cs})$. For sodium, the intercalation is much more difficult so that the first stage compound has never been observed. ${ }^{8}$ However, in spite of its weaker vapour pressure and its lowered electropositive character, lithium leads surprisingly to a pure $\mathrm{LiC}_{6}$ first stage GIC. $5,9,10$

The three alkaline earth metals (calcium, strontium, barium), which are insufficiently electropositive and too little volatile, intercalate very incompletely leading to a two-phase mixture of pristine graphite and first stage GIC of formula $\mathrm{MC}_{6}(\mathrm{M}=\mathrm{Ca}, \mathrm{Sr}$, Ba). ${ }^{11}$

Finally, some lanthanides (samarium, europium, ytterbium) are able to intercalate into graphite with even more difficulties. Indeed, for these metals, the intercalation is exclusively superficial so that the reaction product contains a lot of pristine graphite and a little $\mathrm{MC}_{6}$ first stage GIC (M = Sm, Eu, Yb). ${ }^{12}$

Concerning all these metals, which are little able to intercalate into graphite, an increase of the reaction temperature would enable an easier and more complete intercalation. However, it is not the case, because the too high temperatures provoke the destruction of the graphene layers and the formation of carbides.

It turns out that the first stage $\mathrm{MC}_{8}$ and $\mathrm{MC}_{6}$ binary $\mathrm{GIC}$ show interesting physical properties such as specific magnetic behaviour or superconductivity. Indeed, $\mathrm{MC}_{8}$ GIC show superconducting properties at low temperature, between $0.02 \mathrm{~K}$ and $0.55 \mathrm{~K} .{ }^{13}$ In the case of $\mathrm{MC}_{6}$ compounds, the superconductivity occurs down to $0.065 \mathrm{~K}$ for $\mathrm{BaC}_{6}{ }^{14}, 1.65 \mathrm{~K}$ for $\mathrm{SrC}_{6}{ }^{15}$ and one order of magnitude higher for $\mathrm{CaC}_{6}$ binary GIC with a critical temperature of $11.5 \mathrm{~K} .{ }^{16}$ Among the $\mathrm{MC}_{6}$ compounds with lanthanides, up to now, only $\mathrm{YbC}_{6}$ exhibits 
superconducting properties with a critical temperature of 6.5K. ${ }^{16}$ Other compounds such as $\mathrm{EuC}_{6}$ show remarkable magnetic properties. ${ }^{17}$ In order to obtain strongly reliable results, the study of the physical properties absolutely requires high-quality bulk samples. Unfortunately, they cannot be prepared using the vapour phase method. To overcome the limits of this well-known route, a novel way of synthesis using molten alkali metal-based alloys has been extensively developed in our team. Besides the discovery of several ternary GIC, the use of lithium-based molten alloys allowed the synthesis of two bulk and well characterized binary $\mathrm{MC}_{6}$ compounds: $\mathrm{CaC}_{6}{ }^{18}$ and $\mathrm{EuC}_{6}{ }^{19}$. These syntheses allowed to prepare bulk samples for incontestable and very accurate measurements of superconducting ${ }^{20,21}$ and magnetic properties $^{22}$ respectively. Even more recently, a new synthesis method involving molten salts has been achieved, in order to avoid the presence of inclusions of lithium, which occasionally occurs in some sample synthesised by the molten alloys route. This new method consists in immersing the graphite sample in a liquid eutectic $\mathrm{LiCl}-\mathrm{KCl}$ containing dissolved metal to intercalate. By this way, europium was successfully intercalated leading to a remarkably homogeneous bulk $\mathrm{EuC}_{6} \mathrm{GIC}^{23}$ The present paper deals with the intercalation of three other divalent metals ( $\mathrm{Sr}, \mathrm{Ba}$ and $\mathrm{Yb}$ ) using this new method in order to synthesize pure bulk corresponding binary $\mathrm{MC}_{6}$ allowing future accurate studies of their superconductivity.

\section{Experimental}

Synthesis. The reactions are performed in a glove box under purified argon atmosphere since the reagents are sensitive to air and can be easily oxidized. At first, $\mathrm{LiCl}$ and $\mathrm{KCl}$ salts are individually outgassed under secondary vacuum at $240^{\circ} \mathrm{C}$ for at least 24 hours. The eutectic mixture is then prepared under argon atmosphere using $59.2 \mathrm{~mol} \%$ $\mathrm{LiCl}^{24}$ and $4 \mathrm{~g}$ is weighed and melted in a stainless steel reactor in the $400-450^{\circ} \mathrm{C}$ temperature range. In order to work with a large excess of metal versus graphite, a quantity of 2 to 4 at.\% of the suitable metal $\mathrm{M}(\mathrm{M}=\mathrm{Sr}, \mathrm{Ba}, \mathrm{Yb})$ is added in the molten salt, homogenized by manual stirring. After dissolution of the metal, a single HOPG (Highly Oriented Pyrolytic Graphite) platelet attached to a tungsten support is immersed in the liquid. The reactor is tightly closed with a Swagelok ${ }^{\circledast}$ plug and placed in a metal enclosure under argon in order to safely perform the heat treatment outside the glove box. The whole system is heated in a furnace under suitable synthesis conditions (temperature, duration) in order to reach the thermodynamic equilibrium. Lastly, the sample is extracted in a glove box after melting the reaction medium, and the excess of salt on the surface of the sample is removed by scraping.

The purpose of these experiments is to obtain first stage $\mathrm{MC}_{6} \mathrm{GIC}$ following a formation mechanism for binary compounds already determined by Fauchard et al. ${ }^{25}$ The first step consists in the intercalation of lithium into graphite followed by progressive lithium substitution with the corresponding metal which leads to the desired $\mathrm{MC}_{6}$ compound.

\section{Materials characterization} X-ray diffraction (XRD).

The analyzed samples are synthesized from HOPG platelets. The graphite intercalation compounds thus formed have crystallites whose $\overrightarrow{\mathbf{c}}$ axes are parallel to each other while the ab planes are randomly oriented. Thanks to this texture, the sample behaves as a single crystal along the $\overrightarrow{\mathbf{c}}$ axis and as a powder in the direction parallel to the graphene planes. XRD measurements are carried out with a Bruker D8 Advance diffractometer $(\lambda($ MoK $\alpha 1)=70.926 \mathrm{pm})$. The sample placed in a glass capillary sealed under argon is oriented in the diffractometer in order to record $00 /$ reflections only, by adjusting the ab planes of the sample parallel to the incident beam. By Fourier transform of the $00 /$ structure factors issued from the corresponding diffractogram, the electronic density profile along the $\overrightarrow{\mathbf{c}}$ axis can be calculated, leading to the determination of the stacking sequence of the atomic planes. ${ }^{26}$

Scanning electron microscopy (SEM). SEM is performed with a JEOL JSM6010LA microscope operating at $20 \mathrm{kV}$. Prior to analysis, samples are stuck with carbon tape on a sample holder, under argon atmosphere, and rapidly introduced into the microscope in order to limit their air exposure.

Ion beam analyses. Ion beam analyses have been used in order to probe the composition of the samples and their homogeneity, in-depth (several tens of microns) as well as laterally. They are based on beam-matter interactions, i.e. in this study between a ${ }^{1} \mathrm{H}^{+}$proton ion beam of $3 \mathrm{MeV}$ and the target consisting in the GIC sample, the detector being placed at an angle of $170^{\circ}$ from the beam axis. For $\mathrm{Cl}, \mathrm{K}, \mathrm{Sr}, \mathrm{Ba}$ and $\mathrm{Yb}$, no nuclear reactions occur between the target nuclei and the proton ion beam. The only interactions taken into account are Rutherford Back Scattering (RBS) ones, easily calculated for these heavy elements. For carbon, in these experimental conditions, we use the advantage to get an enhanced beam-matter interaction and interpret the information obtained from non-Rutherford Back Scattering interactions (called PES for Proton Enhanced Scattering). Finally, in the case of lithium, we have considered as cross-sections both PES interaction and nuclear reaction between the ion beam and lithium. Consequently, one recorded spectrum (with a resolution of few micrometers due to the $4 \times 4 \mu \mathrm{m}^{2}$ ion beam size) contains all information concerning the aforementioned elements, on $200 \times 200 \mu \mathrm{m}^{2}$ square maps. The collected data are then interpreted using the SimNRA software ${ }^{27}$, leading to the description of a sample composed of several layers of different composition and various thickness. The final simulated spectrum is then composed of the superimposed contributions of all the interactions, with each isotope of each element of each sublayer of the defined solid.

\section{Magnetism.}

The magnetic behavior of $\mathrm{YbC}_{6}$ was checked by dc magnetometry through a commercial dc-squid (MPMS2 by Quantum Device). Low temperature dc susceptibility was performed by applying a magnetic field of $1 \mathrm{mT}$. 


\section{Results and discussion}

\section{Intercalation of alkaline-earth metals}

Syntheses were carried out at $450^{\circ} \mathrm{C}$ during 6 days for the intercalation of strontium and barium. Fig. 1 shows the diffractograms for these two samples. The patterns of Fig. 1a and $\mathbf{1 b}$ show intense and individualized $00 /$ reflections. The latter correspond respectively to $\mathrm{SrC}_{6}$ and $\mathrm{BaC}_{6}$ first stage binary $\mathrm{GIC}$ whose repeat distances $I_{C}$ are 494 and 525 pm respectively.
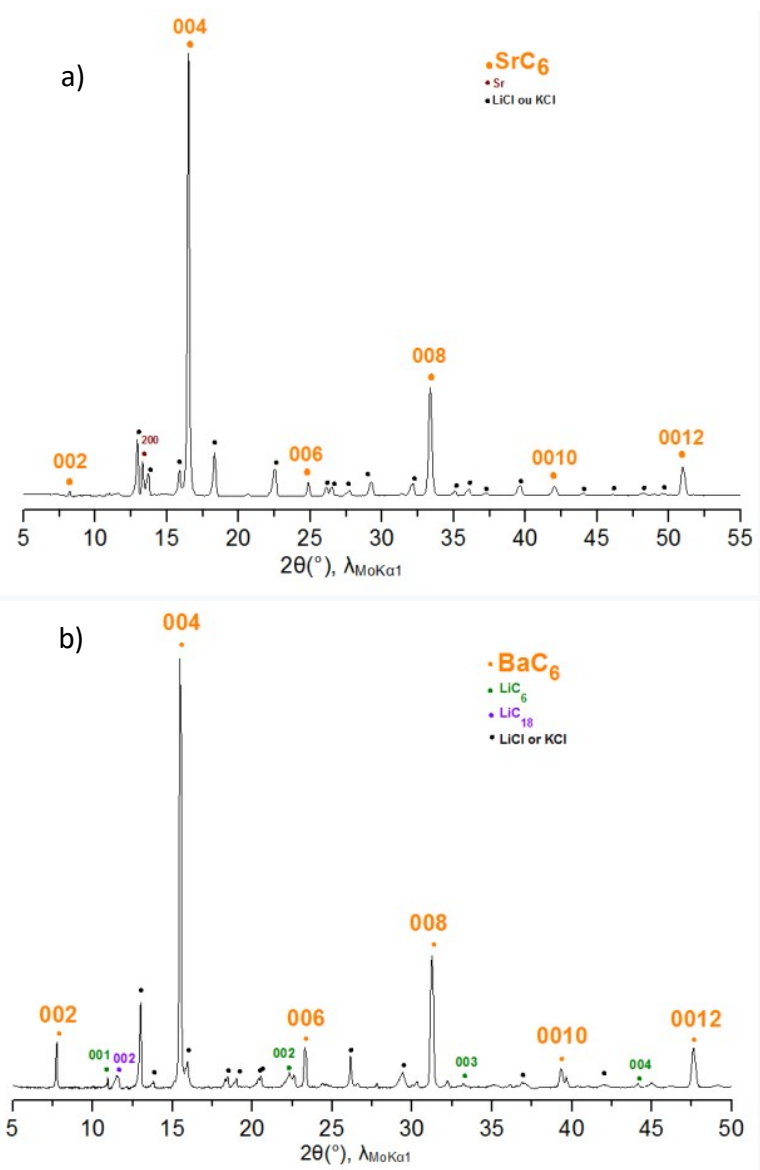

Fig.1 00/ X-ray diffraction patterns $\left(\lambda_{\text {MoK } \alpha 1}=70.926 \mathrm{pm}\right)$ of the first stage $\mathrm{GIC}$ a) $\mathrm{SrC}_{6}\left(\mathrm{I}_{\mathrm{C}}=494 \mathrm{pm}\right)$ and b) $\mathrm{BaC}_{6}\left(\mathrm{I}_{\mathrm{C}}=525 \mathrm{pm}\right)$

The reflections of pristine graphite are not observed and those of first stage GIC are predominant indicating that a total intercalation has taken place. Both GIC crystallize in the same hexagonal structure (space group $P 6_{3} / m m c$ with the $A \alpha A \beta$ stacking sequence along the $\overrightarrow{\mathbf{c}}$ axis). ${ }^{11}$

In both cases, small reflections are observed referring mainly to the superficial presence of $\mathrm{LiCl}$ and $\mathrm{KCl}$ salts. In the case of the $\mathrm{LiCl}-\mathrm{KCl} / \mathrm{Sr}$ system, some metal inclusions of strontium are detected, in agreement with diffraction peak at $2 \theta=13.3^{\circ}$. In the case of barium, the bulk intercalation of this metal is evidenced regarding the presence of the $00 /$ reflections corresponding to a $1^{\text {st }}$ stage Ba-GIC. Only weak reflections of stage- 1 and stage- 2 Li-GIC are recorded. This result is in agreement with the mechanism already determined by Fauchard et al. ${ }^{25}$
Ion beam analyses using nuclear microprobe instrument has shown all its interest for the study of the chemical composition of GIC. ${ }^{28}$ It has been used in this study to analyze the chemical composition of Sr-based, Ba-based and Yb-based GIC. Ion beam analysis performed on the $\mathrm{Sr}$-based GIC is given Fig.2.

The presence of potassium and chlorine on the spectrum is due to residual salt not perfectly evacuated from the surface of the sample by mechanical sweeping. Oxygen also visible is due to contamination during the fast transfer of the sample into the nuclear microprobe device. The presence of $\mathrm{K}, \mathrm{Cl}$ and $\mathrm{O}$ as peaks implies their presence only on the surface of the probed sample. Concerning individual contributions of $\mathrm{Sr}$ and $\mathrm{C}$, they can be well fitted on small areas only considering the expected $\mathrm{SrC}_{6}$ stoichiometry.

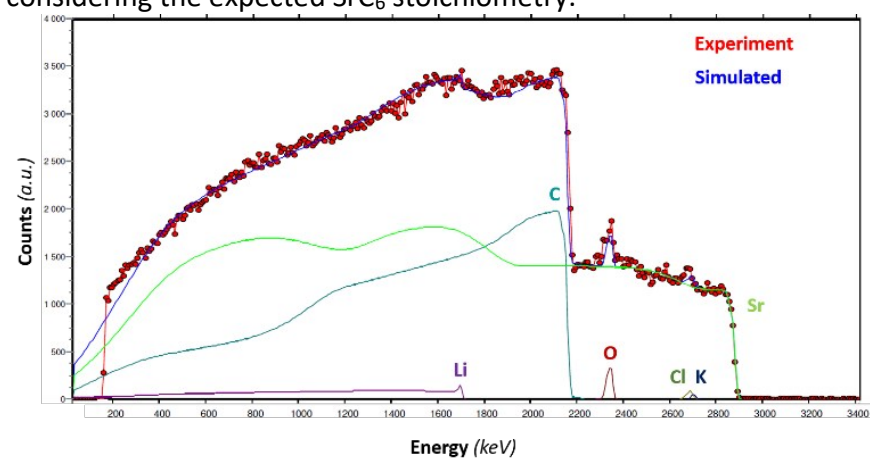

Fig.2. Recorded RBS/NRA spectrum of the Sr-based GIC synthesized by the molten salts method and its simulation. Each individual elementary contribution to the simulation is drawn. Red spots: experiment; blue solid line: simulation.

This statement reinforces the fact that strontium is intercalated into the bulk of graphite. Finally, it should be noted that residual signal of lithium is detected due to nuclear reactions. It corresponds to about 4 atomic percent of lithium (not detected by X-ray diffraction), homogeneously distributed in the $\mathrm{SrC}_{6}$ sample. Considering the reaction mechanism published earlier concerning the synthesis in molten salts ${ }^{25}$, we assume that lithium has not been totally evacuated from van der Waals's gaps when $\mathrm{Sr}$ atoms substituted $\mathrm{Li}$ ones. This explains why the sample cannot be fitted as a unique slice of pure $\mathrm{SrC}_{6}$ but is considered as several small areas that can be individually fitted as $\mathrm{SrC}_{6}$ GIC containing residual lithium. Magnetic measurements can be performed in order to probe the exact critical temperature of bulk $\mathrm{SrC}_{6}$ but this expected low temperature necessitates more than a classical cryogenic device.

Then, we carried out equivalent ion beam analyses on Ba-based GIC. A representative spectrum is given Fig.3. It immediately appears that the spectrum is more "noisy ", with several irregularities that make it difficult to model the possible presence of $\mathrm{K}, \mathrm{Cl}$ or $\mathrm{O}$ on the surface of the sample. In fact, this spectrum can roughly be fitted considering several $\mathrm{BaC}_{6}$ slices surrounded with lithium veins. This results with overconcentration in lithium at given depth, as well as Ba and C depletions at the same location. Such $\mathrm{Li}$ veins have already been observed before considering GIC synthesized using solid-liquid reactions. ${ }^{29}$ Such an observation cannot be done by XRD, that is why this complementary approach (XRD and ion beam studies) should be 
systematically realized to obtain a complete description of GIC synthesized by solid-liquid reactions.

It is now necessary to probe the superconducting behavior of the soprepared $\mathrm{BaC}_{6}$ but the expected $T_{C}$ once again needs specific measurements and precautions.

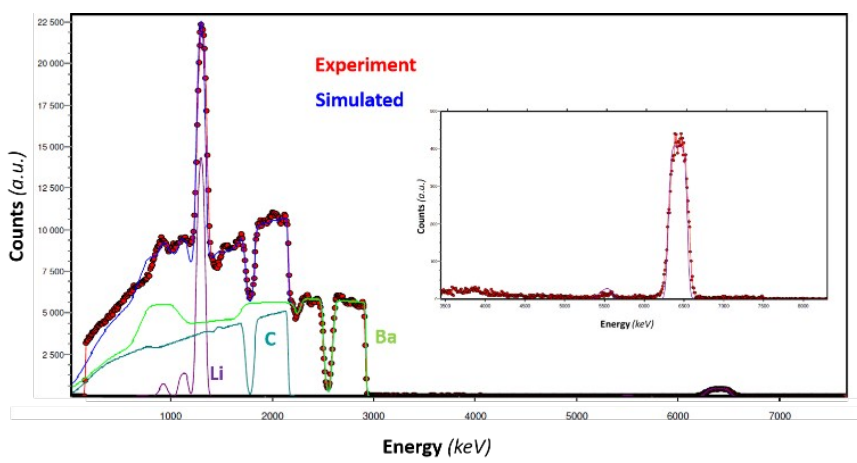

Fig.3. Recorded RBS/NRA spectrum of the Ba-based GIC synthesized by the molten salts method and its simulation. Each individual elementary contribution to the simulation is drawn. Red spots: experiment; blue solid line: simulation. The inset is focused on the ${ }^{7} \mathrm{Li}$ nuclear reaction energy range.

\section{Intercalation of ytterbium.}

The first attempts to synthesize lanthanide-graphite intercalation compounds were made by El Makrini et al. in 1980. ${ }^{12}$ Some reactions lead to $\mathrm{EuC}_{6}, \mathrm{YbC}_{6}, \mathrm{SmC}_{6}$ and $\mathrm{TmC}_{6}$ only superficially intercalated compounds. Later, fully intercalated Eu-GIC has been synthesized. ${ }^{19,23}$ Several tries were made to obtain a bulk $\mathrm{YbC}_{6}$ intercalation compound, however attempts reported in the literature remained unsuccessful. ${ }^{16}$

Varying experimental parameters, ytterbium intercalation has been performed using the molten salt method. Reactions are realized using 2-4 at.\% of $\mathrm{Yb}$, the temperature is chosen in the $405-425^{\circ} \mathrm{C}$ range for duration between 6 and 10 days.

The diffractogram (Fig.4.) evidences the presence of first stage $\mathrm{YbC}_{6}$ in a Li-GIC (first and second stages) matrix. Indeed, four $00 /$ reflections of $\mathrm{YbC}_{6}$ are visible, indicating a promising result. Whatever is the considered reaction duration, the amount of $\mathrm{YbC}_{6}$ in the final sample remains constant. This observation is in total agreement with the phenomenon noted in the $\mathrm{LiCl}-\mathrm{KCl} / \mathrm{Ca}$ system. ${ }^{25}$

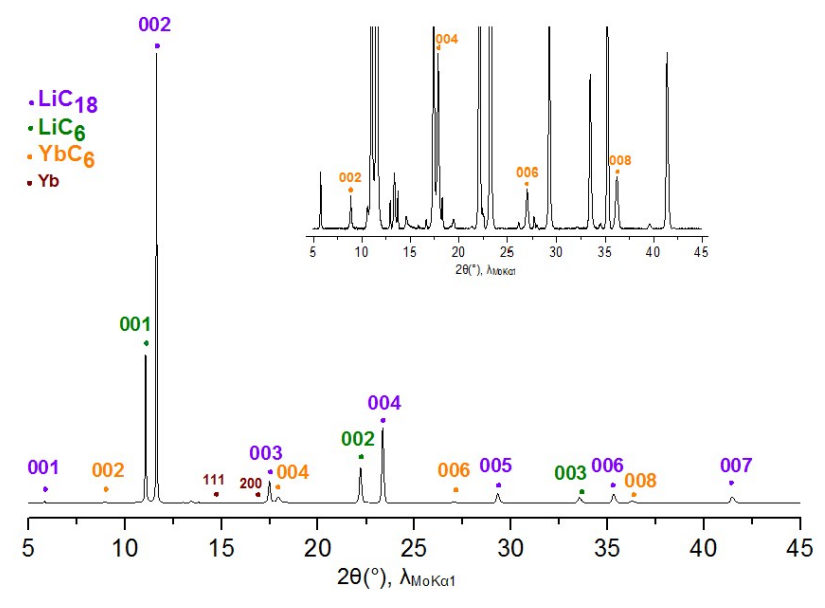

Fig.4. 00/ X-ray diffraction pattern $\left(\lambda_{\text {MoKa1 }}=70.926 \mathrm{pm}\right)$ $\mathrm{YbC}_{6}$-containing sample. The zoom is referred to $\mathrm{YbC}_{6}$ reflections

Fig. 5 presents $\mathrm{SEM}$ micrographs of the $\mathrm{YbC}_{6}$-containing sample with different magnifications, and corresponding energy dispersive X-ray spectroscopy (EDXS) results collected on two different zones of the sample.

The micrograph 5.a) shows the entire sample and reveals the presence of residual surface impurities providing from the reaction medium. The micrograph 5.b) is collected on the edge of the sample (backscattered electrons imaging) and shows dark zones inward the sample and light zones at the extreme border, which indicates a chemical heterogeneity. Indeed, EDX spectrum on dark zone 1 (Fig.5.c) mainly reveals the presence of carbon with traces of chlorine and potassium provided by the eutectic medium, and spectrum Fig.5.d shows carbon and ytterbium only. Given the absence of pristine graphite on the corresponding diffractogram, carbon is necessarily associated to another element. Then, carbon evidenced by EDXS on zone 1 actually corresponds to Li-based GIC, as lithium is too light to be detected by this method. EDXS results on zone 2 indicate the presence of carbon and ytterbium in agreement with the likely presence of $\mathrm{YbC}_{6}$ evidenced by $00 / \mathrm{X}$-ray diffraction. The intercalation of ytterbium is incomplete and localized on the edges of the sample.
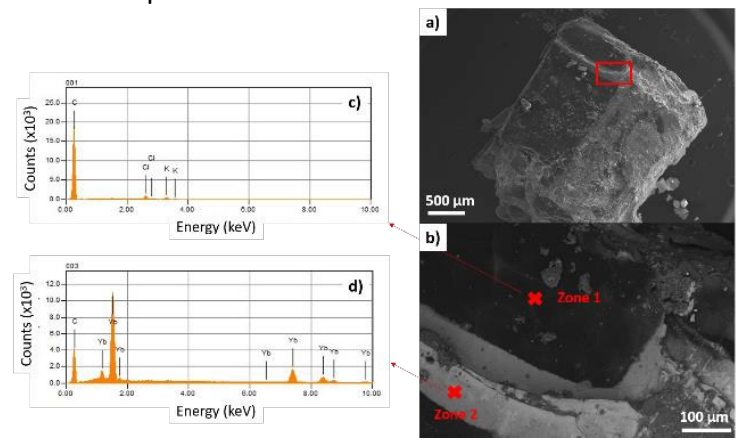

Fig. 5. a) SEM micrograph (secondary electrons imaging) of the $\mathrm{YbC}_{6}$-containing sample b) SEM micrograph (backscattered electrons imaging) of the framed zone c) EDX spectrum on zone 1 d) EDX spectrum on zone 2. 
XRD and SEM experiments have pointed out the evidence of superficial intercalation of ytterbium into graphite using a reaction in molten salts medium. Ion beam analyses confirm these results. Indeed, the study of a probed area considering the region of interest of ytterbium reveals on the south-west quarter the presence of a Yb-rich area (Fig.6.).

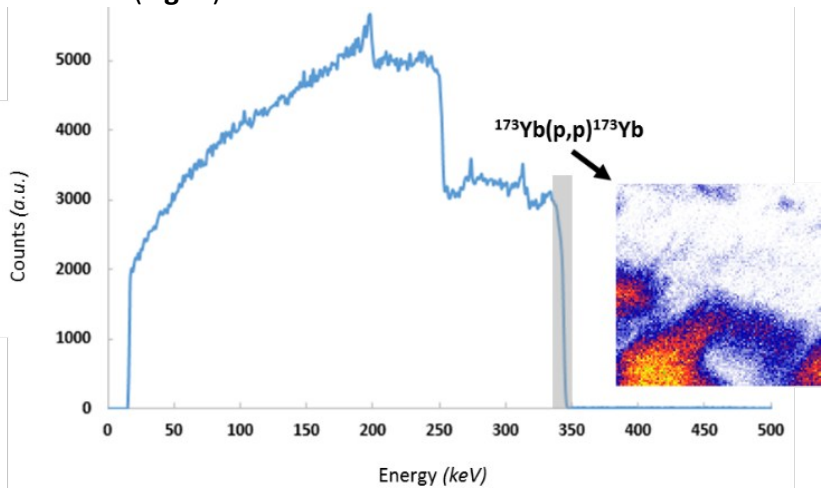

Fig 6. Spectrum of the $\mathrm{YbC}_{6}$-containing sample. The grey area indicates the energy windows considered to draw contrast maps of ytterbium.

This region represents less than $5 \%$ of the total surface of the map. Experimental data corresponding to this part of the map have been extracted and fitted (Fig.7). It clearly appears that this fraction of the sample corresponds to a bulk $\mathrm{YbC}_{6}$ phase, as the experimental spectrum is well restituted considering a unique slice of this later composition. So, here we confirm the intercalation of ytterbium into graphite in-depth however only on small fractions of the sample, and probably located closed to edges of graphite.

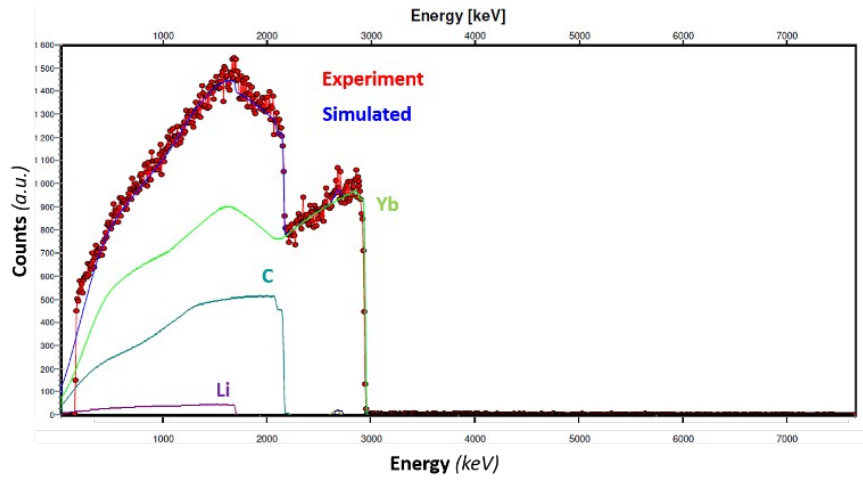

Fig.7. Recorded RBS/NRA spectrum of the $\mathrm{YbC}_{6}$-containing sample synthesized by the molten salts method and its simulation. Each individual elementary contribution to the simulation is drawn. Red spots: experiment; blue solid line: simulation.

In figure 8 we plot the zero field cooled (ZFC) low temperature behaviour of the dc mass susceptibility $\chi_{m}$ measured with an applied magnetic field of $1 \mathrm{mT}$, that is well below the first critical field $\sim 50 \mathrm{mT}$ expected for this compound. ${ }^{16}$ The plotted data result from the subtraction $\chi_{m}(T)-\chi_{m}\left(T>T_{C}\right)$ in order to remove a small positive offset due to a spurious ferromagnetic-like impurity probably present in the sample-holder. We note several interesting features: $i$ ) a clear superconducting onset is present at $6 \mathrm{~K}$, i.e. very near to the expected transition temperature fixed at $6.5 \mathrm{~K} .{ }^{16}$ ii) A shoulder is present at about $3.9 \mathrm{~K}$ below which $\chi_{\mathrm{m}}$ decreases quite sharply. This likely indicates the presence of sample regions with reduced $\mathrm{T}_{\mathrm{C}}$ but with a stronger diamagnetic response. iii) We could estimate an upper bound for the superconducting shielding fraction of about $10 \%$ at $2 \mathrm{~K}$ providing the assumption that the sample could be homogenously intercalated. By concluding, this measure confirms the inhomogeneous nature of the intercalation in the present sample. Nevertheless, encouraging traces of superconductivity were found in the expected temperature interval.

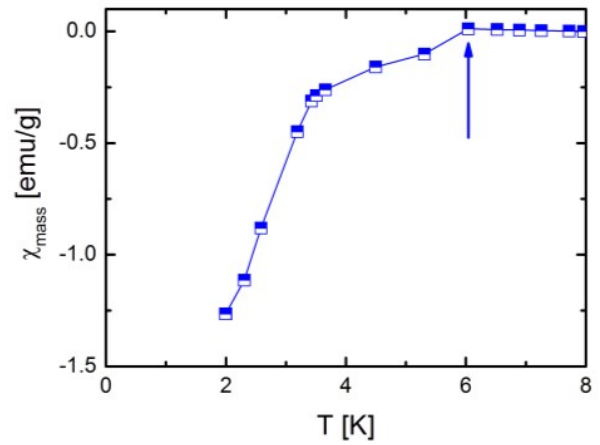

Fig.8. Low temperature ZFC mass susceptibility $\chi_{m}$ of the $\mathrm{YbC}_{6}$-containing sample taken under an external field of $1 \mathrm{mT}$. The arrow indicates the superconducting critical temperature $T_{c}$.

\section{Conclusion}

This paper deals with the possibility to realize solid-liquid reactions in a $\mathrm{LiCl}-\mathrm{KCl}$ molten salt medium in order to perform intercalation of alkaline-earth and lanthanide metals into graphite, , elements which are hardly intercalated alone using the classical vapour phase technique.

Similarities can be stated compared to another solid-liquid reaction in Li-based molten alloys used elsewhere for the preparation of GIC. For both ways of synthesis, it has been shown that lithium (present in the former alloy or formed in situ in the molten salts) is first intercalated into graphite, allowing to spread apart graphene sheets. Then, the substitution of lithium with the other metallic element present in the reactive medium occurs, leading to bulk first stage GIC. In this study, it is shown that a bulk $\mathrm{SrC}_{6}$ compound $\left(\mathrm{I}_{\mathrm{C}}=494 \mathrm{pm}\right)$ never successfully synthesized before can be prepared using the $\mathrm{LiCl}-\mathrm{KCl} / \mathrm{Sr}$ mixture. This result highlights the strong interest of our procedure. XRD experiments agree with a bulk intercalation compound, however ion beam analyses show that some efforts are still necessary in order to determine the best conditions for the preparation of a perfectly homogeneous compound. 
We have also successfully prepared a bulk $1^{\text {st }}$ stage $\mathrm{BaC}_{6} \mathrm{GIC}$ $\left(I_{C}=525 \mathrm{pm}\right)$. XRD experiments are in agreement with this conclusion, and once again ion beam analyses appear as a powerful complementary tool as these measurements show the presence of lithium veins at different depths, separating $\mathrm{BaC}_{6}$ homogeneous domains.

Finally, the study of the $\mathrm{LiCl}-\mathrm{KCl} / \mathrm{Yb}$ system has been undertaken, considering that $\mathrm{YbC}_{6}$ is especially interesting regarding its relatively high $T_{C}$ value $(6.5 \mathrm{~K})$. We revealed the obtaining of a polyphasic sample however containing small $\mathrm{YbC}_{6}$ areas in a Li-based GIC matrix (Li-GIC of first and second stage). This constitutes a promising result concerning the intercalation of ytterbium into graphite and trials are currently carried out to obtain a bulk and pure $\mathrm{YbC}_{6}$ sample.

\section{Conflicts of interest}

There are no conflicts to declare.

\section{Acknowledgements}

The acknowledgements come at the end of an article after the conclusions and before the notes and references.

\section{References}

1 C. Mauguin, Bull. Soc. Chim. Fr., 1926, 49, 32-61

2 S. Cahen, L. Speyer, P. Lagrange and C. Hérold, Eur. J. Inorg. Chem., 2019, 4798-4806

3 A. Hérold, Mater. Sci. Eng., 1977, 31, 1-16

4 N. Daumas and A. Hérold, C. R. Acad. Sc. Paris C, 1969, 268, 373-375

5 A. Hérold, Bull. Soc. Chim. Fr., 1955, 187, 999-1012

6 K. Fredenhagen and G. Cadenbach, Z. Anorg. Allgem. Chem., 1926, 158, 249-263

7 W. Rüdorff and E. Schulze, Z. Anorg. Allgem. Chem., 1956, 277, 156-171

8 A. Métrot, D. Guérard, D. Billaud and A. Hérold, Synth. Met., 1980, 1, 363-369

9 M. Bagouin, D. Guérard and A. Hérold, C. R. Acad. Sc. Paris, 1966, 262, 557-559

10 D. Guérard and A. Hérold, Carbon, 1975, 13, 337-345

11 D. Guérard, M. Chaabouni, P. Lagrange, M. El Makrini and A. Hérold, Carbon, 1980, 18, 257-264

12 M. El Makrini, D. Guérard, P. Lagrange and A. Hérold, Carbon, 1980, 18, 203-209

13 N.B. Hannay, T.H. Geballe, B.T. Matthias, K. Andres, P. Schmidt and D. Mac Nair, Phys. Rev. Lett. 1965, 14, 225-226

14 S. Heguri, N. Kawade, T. Fujisawa, A. Yamaguchi, A. Sumiyama, K. Tanigaki and M. Kobayashi, Phys Rev Lett., 2015, 114(24), 247201

15 J.S. Kim, L. Boeri, J.R. O'Brien, F.S. Razavi and R.K. Kremer, Phys. Rev. Lett., 2007, 99, 027001

16 T.E. Weller, M. Ellerby, S.S. Saxena, R.P. Smith and N.T. Skipper, Nature Phys., 2005, 1, 39-41
17 H. Suematsu, K. Ohmatsu, T. Sakakibara, M. Date and M. Suzuki, Synth. Met., 1983, 8(1-2), 23-30

18 N. Emery, C. Hérold and P. Lagrange, J. Solid State Chem. 2005, 178, 2947-2952

19 H. Rida, S. Cahen, C. Hérold and P. Lagrange, Carbon, 2010, 48, 3190-3195

20 N. Emery, C. Hérold, M. d'Astuto, V. Garcia, C. Bellin, J.F. Marêché, P. Lagrange and G. Loupias, Phys. Rev. Lett., 2005, 95, 087003

21 A. Gauzzi, S. Takashima, N. Takeshita, C. Terakura, H. Takagi, N. Emery, C. Hérold, P. Lagrange and G. Loupias, Phys. Rev. Lett., 2007, 98, 067002

22 G. Lamura, T. Shiroka, S. Cahen, H. Rida, N. Emery, J.F. Marêché, P. Lagrange and C. Hérold, Carbon, 2012, 50, 39954001

23 M. Bolmont, S. Cahen, M. Fauchard, R. Guillot, G. Medjahdi, P. Berger, G. Lamura, P. Lagrange and C. Hérold, Carbon, 2018, 133, 379-383

24 A.S. Basin, A.B. Kaplun, A.B. Meshalkin and N.F. Uvarov, Inorg. Chem., 2008, 53, 1509-1511

25 M. Fauchard, S. Cahen, M. Bolmont, G. Medjahdi, P. Lagrange and C. Hérold, Carbon, 2019, 144, 171-176

26 S. Y. Leung, M. S. Dresselhaus, C. Underhill, T. Krapchev, G. Dresselhaus and B. J. Wuensch, Phys. Rev. B, 1981, 24, 35053518

27 M. Mayer, Proceedings, $15^{\text {th }}$ International Conference on the Application of Accelerators in Research and Industry, 1999, 475, 541-544

28 P. Berger, S. Pruvost, C. Hérold and P. Lagrange, Carbon, 2004, 42, 2049-2056

29 P. Berger, S. Pruvost, C. Hérold and P. Lagrange, Nucl. Instrum. Methods B, 2004, 219-220, 1005-1009 